\title{
Hematoma renal en paciente monorreno
}

\author{
Luis Basco Prado - Silvia Fariñas Rodríguez ${ }^{1}$
}

Diplomado en Enfermería. Licenciado en Antropología. SCIAS. UCI Hospital De Barcelona. Profesor Facultad Enfermería de la Universidad de Barcelona. Departamento Enfermería fundamental y Médico-Quirúrgica ${ }^{1}$ Diplomada en Enfermería. Servicio UCI y Urgencias Clínica CIMA

\section{Introducción}

Descrito en 1856 por Wunderlich, el hematoma perirrenal espontáneo es una patología infrecuente, en la que a pesar de llegar al diagnóstico de una manera relativamente sencilla con estudios imagen, el poder determinar la etiología representa un reto y es motivo de múltiples estudios y procedimientos invasivos, algunas veces sin poder llegar a esclarecer la causa ${ }^{1}$. Los hematomas renales y esplénicos están asociados con frecuencia a traumatismos abdominales y más raramente se deben a alteraciones de la coagulación e infecciones. Otras causas menos frecuentes son procesos inflamatorios, diátesis sanguínea, enfermedad poliquística, aneurismas, malformaciones arteriovenosas, infarto e idiopáticos ${ }^{2}$.

El área subcapsular del riñón es un espacio potencial donde se pueden acumular los fluidos causando compresión al parénquima renal. Este tipo de fluidos puede consistir en colecciones de pus, sangre, linfa, exudado o trasudado ${ }^{3}$. Pacientes con traumatismos importantes, quemaduras extensas, operaciones quirúrgicas complicadas, procesos ginecoobstétricos graves, enfermedades infecciosas severas e intoxicaciones agudas, tienen alto riesgo para desarrollar Fracaso Renal Aguda4.

Correspondencia:

Luis Basco Prado

Avenida Diagonal 660

08034 Barcelona

E-mail: luisbasco@ub.edu
Las técnicas continuas de reemplazo renal (TCRR) constituyen una de las principales técnicas y tratamientos de soporte de los pacientes con Fracaso renal Agudo (FRA). La incidencia de FRA en pacientes críticos ingresados en las unidades de cuidados intensivos (UCI) es claramente superior al resto de la población hospitalaria y puede llegar a afectar a más del 35\% de estos pacientes ${ }^{5,6}$.

Presentamos a continuación un caso clínico de hematoma renal no traumático con consecuencias clínicas trascendentes.

\section{CASO CLÍNICO}

Paciente de 65 años que acude al servicio de urgencias por cuadro de dolor lumbar intenso irradiado a genitales de forma brusca y espontánea. Dolor lumbar intenso con hematuria y repercusión en estado general del paciente. No refiere antecedente traumático previo.

\section{Antecedentes Patológicos:}

- HTA

- Dislipemia.

- Cardiopatía isquémica tratada con tres stents coronarios.

- Valvulopatía Aórtica.

- By Pass Aorto-Coronario y Recambio Valvular Aórtico en 2008.

- Monorreno por Adenocarcioma renal tratado con nefrectomía derecha en 2003.

- Medicación habitual en casa: Acenocumarol. 
El paciente está consciente y orientado (Glasgow coma Scale 15). Normohidratado y normocoloreado. Abdomen doloroso de forma difusa pero sin defensa ni peritonismo. No se aprecian masas ni megalias. Ileoparalítico. Radriografía abdominal sin alteraciones.

Analítica: 16.000 leucocitos $/ \mathrm{mm}^{3}$, Creatinina $191 \mu \mathrm{mol} / \mathrm{L}$,

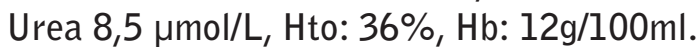

Se solicita Ecografía renal y Tomografía Axial Computerizada (TAC), hallándose extenso sangrado subcapsular renal y perirrenal izquierdo, con discreta extensión a los espacios pararrenales adyacentes. No se identifica el origen del sangrado pero en el doppler renal existe la sospecha de sangrado activo hacia el espacio subcapsular. Presencia de pequeña litiasis en el seno renal. La vía excretora no está dilatada.

Se realiza consulta con el Servicio de Urología que a su vez contacta con la Unidad de Cuidados Intensivos para el ingreso del paciente, puesto que se considera en primera instancia la reversión del cuadro clínico antes de realizar cualquier tipo de maniobra diagnósticoterapéutica invasiva.

El paciente ingresa en la Unidad de Cuidados SemiIntensivos para el control de los síntomas y monitorización de la hemostasia y función renal.

Al ingreso en la unidada se realiza de nuevo analítica control que muestra $\mathrm{Hb}: 9,8 \%$, Hto: $29,1 \mathrm{~g} / 100 \mathrm{ml}$, Leucocitos: $15.200 / \mathrm{mm}^{3}$, TTPA: 32s.

La analítica 8 horas después muestra una anemización severa ( $\mathrm{Hb}: 6,6 \%$ ), creatinina: $400 \mathrm{mmol} / \mathrm{l}$, no hiperpotasemia, TP> 45\%, mostrando al paciente oligoanúrico, hemodinámicamente inestable, con necesidades crecientes de oxigenación, dolor a la palpación en flanco izquierdo, nauseoso y con algún vómito.

Se procede a la optimización de las medidas de recuperación de diuresis; corrección de la volemia y de la anemia + Bomba de Perfusión Continua (BPC) de noradrenalina y furosemida en bolus, sin demasiada efectividad. Se plantea la posibilidad de iniciar Técnicas Continuas Reemplazo Renal (TCRR) ya que dichas técnicas constituyen el principal tratamiento de soporte de los pacientes con FRA y Disfunción Multiorgánica (DM0), aunque la mortalidad hospitalaria sigue siendo muy elevada (alrededor del 60-80\%) ${ }^{7,8}$.
A pesar de décadas de experiencia en el uso de técnicas de hemodiálisis para el soporte en el FRA, todavía existen dudas acerca del momento óptimo de comenzarlas, de la mejor técnica por utilizar y de la dosis más adecuada?.

Dado el cuadro clínico del paciente, no existen posibilidades de drenaje percutáneo ni descompresión quirúrgica, planteándose la posibilidad de una nefrectomía en un segundo tiempo, ya que esto condicionaría la necesidad de sustitución renal de por vida. Durante el segundo día de ingreso se suministran concentrados de hematíes y plasma por lo que la anemización progresiva que mantenía el paciente parece estar controlada. La hemodinámia con las aminas vasoactivas parece estabilizarse pero la oligoanuria persiste obligando a instaurar una BPC de furosemida. La función respiratoria continúa cayendo, necesitándose cada vez más aporte de 02, llegando a instaurarse finalmente una Ventilación Mecánica No Invasiva (VMNI).

A las 24 horas (tercer día de ingreso) de instauración de las terapias (optimización de precarga con volumen, tensión arterial con aminas vasoactivas y presión de perfusión renal con diuréticos) el paciente muestra unas constantes hemodinámicas y renales dentro de los parámetros de la normalidad. Durante las siguientes 24 horas (cuarto día de ingreso) el paciente parece mantenerse estable hemodinámicamente aunque con el inconveniente de que vuelve a quedar anúrico. No se evidencia resangrado. Ante la posibilidad de un nuevo sangrado o de una trombosis de arteria renal se solicita TAC y ecodoppler renal.

La ecografía renal muestra que el hematoma subcapsular y perirrenal izquierdo ha aumentado discretamente de tamaño, confirmando la sospecha que existía en la ecografía previa de persistencia de sangrado activo. EI doppler renal no evidencia flujo en el hematoma por lo que se estima de que el sangrado ha cesado. Muestra también que existe cierto flujo renal y venoso en el interior del riñón. Existe discreta cantidad de líquido libre perihepático. EITAC refleja derrame pleural biblasal en cantidad leve-moderada con atelectasia de ambas bases pulmonares, no presentes en el TAC previo.

En Gasometría Arterial (GSA) acidosis respiratoria e hipoxemia debido a atelectasia bibasal secundaria a derrame pleural bilateral. Se continúa con sesiones de VMNI. 
El paciente durante la noche (cuarto día ingreso) comienza con agitación psicomotriz y desorientación, mostrando su negativa a recibir oxigenación mediante la VMNI. Dado el cuadro clínico del paciente y la actual situación motiva a la sedoanalgesia del paciente y la intubación orotraqueal (IOT) de forma reglada. Maniobra de IOT extraordinariamente laboriosa y de gran dificultad por encontrar un stop más allá de las cuerdas vocales (posible granuloma subglótico) que no deja pasar el tubo endotraqueal (TET). Finalmente se consigue colocar un TET de 7,5. Se atribuye la dificultad de la intubación a que el paciente hace dos años había sido traqueostomizado de forma percutánea. Estas dificultades obligan a trasladar al paciente a la Unidad de Cuidados Intensivos.

Con todas las medidas de soporte se ha conseguido por el momento una diuresis suficiente como para no realizar ninguna sesión de hemodiálisis. Durante el sexto día de ingreso obtenemos diuresis de casi $1 \mathrm{ml} /$ $\mathrm{kg} / \mathrm{h}$ pero con creatininas de $700 \mathrm{mmol} / \mathrm{l}$, normovole$\mathrm{mia}$, no hiperpotasemia. Se evidencia ligera febrícula que se interpreta en el contexto del gran hematoma del retroperitoneo.

Se cursa cultivo de secreciones debido a la incipiente broncorrea del paciente y todo el tiempo de maniobras en vía aérea superior y contexto clínico del paciente. El cultivo muestra una infección por pseudomona aeruginosa que alerta sobre el peor pronóstico del paciente ya que la neumonía bacteriana es la infección intrahospitalaria más frecuente en los enfermos ingresados en la UCI y con mayor mortalidad que puede ser extrema en aquellos con el síndrome de distrés respiratorio agudo (SDRA) ${ }^{10}$.

Valoración diaria de posibilidad de TCRR. La hemofiltración y hemodiafiltración continua se consideran técnicas útiles en pacientes que cursan con complicaciones sépticas graves ${ }^{3}$.

Se inicia nutrición enteral a ritmo de $1000 \mathrm{~mL} / 24 \mathrm{~h}$. A las $12 \mathrm{~h}$ se detiene por retención masiva $(700 \mathrm{~mL})$ y se plantea el inicio de nutrición parenteral (NP).

El paciente realiza una buena diuresis total (2000 $\mathrm{mL}$ ). Las cifras de creatinina descienden ligeramente, no se muestra hiperpotasémico, no acidótico, sin ningún signo de sangrado activo por lo que de momento continúa no siendo candidato a las terapias continuas de reemplazo renal (TCRR).
En las últimas $24 \mathrm{~h}$ aparece condensación unilateral en el hemitórax derecho causando SDRA con I02 de 60. Supuestamente neumonía aspirativa de inicio durante las maniobras de IOT y /o al episodio de aspiración de gran contenido dieta enteral.

Se cursa cultivo secreciones y se empieza empíricamente tratamiento antibiótico de amplio espectro ajustado a su función renal. El cultivo muestra una infección por Klebsiella.

Se inicia BPC de relajantes musculares con atracurio por tal de adaptar mejor al paciente a la VM. El paciente se muestra más hipotenso, por lo que hay que aumentar aminas vasoactivas $y$, oligurico que evoluciona a anuria. Respecto a la función ventilatoría las siguientes $24 \mathrm{~h}$ el paciente parece mantenerse estable con todas las terapias administradas. Reaparece la febrícula hasta alcanzar los $38,5^{\circ} \mathrm{C}$ que se acompaña de acidosis metabólica no láctica, fibrilación auricular con frecuencias ventriculares medias de 115-125x' refractaria a un bolus de amiodarona por lo que se realiza cardioversión hasta en tres ocasiones (70-100$150 \mathrm{~J}$ ) no obteniendo de nuevo ritmo sinual por lo que se pauta de amiodarona.

Dada la situación clínica del paciente, finalmente se decide canalizar vía con catéter para posible instauración de TCRR en la modalidad HDFVVC. Se mantiene la situación de hipertermia (Ilegando hasta $41,3^{\circ} \mathrm{C}$ ). En todo este contexto se decide el inicio de TCRR con HDFVVC como técnica de elección ya que al implementarse de un modo lento y continuo, evitan algunas complicaciones de la hemodiálisis convencional (HC) cuando son aplicadas al paciente crítico con fracaso renal agudo (FRA). Las técnicas continuas aseguran una mayor estabilidad hemodinámica y evitan cambios bruscos en la volemia y en las concentraciones de electrólitos y urea. Además, facilitan una reposición hidroelectrolítica más ajustada y una administración de medicación y nutrición enteral o parenteral total más segura ${ }^{11}$. La HDFVVC, ofrece mediante difusión y convección, el aclaramiento de solutos de peso molecular pequeño y mediano, teniendo la opción de extracción de líquido del paciente por ultrafiltración, manejando flujos sanguíneos con menor velocidad que la hemodiálisis, por lo que ofrece mayor estabilidad hemodinámica; además de la capacidad de lograr la depuración de mediadores pro-inflamatorios, que son la piedra angular en el síndrome de respuesta 
inflamatoria sistémica y componente esencial en la fisiopatología de la sepsis y la disfunción orgánica múltiple ${ }^{12}$.

En las 6 horas post inicio TCRR, el paciente se muestra hemodinámicamente más estable, función renal conservada y normotérmico. En las siguientes 12 horas (octavo día de ingreso), la función ventilatoria del paciente empeora. Severa acidosis metabólica y diselectrolitemia (hiperpotasemia 6,3) que desencadena en trastornos del ritmo y conducción eléctrica cardíaca con bradicardia extrema. $\mathrm{Ph}: 7,1$ con Exceso Base (-EB) de -10 de causa respiratoria que no compensa con medidas de intento de normoventilación (Volumen Minuto de 13 litros por minuto y Presión Control rozando el barotrauma). El cuadro desemboca en medidas de resucitación que finalmente no son efectivas.

\section{Conclusión}

El hematoma renal de origen no traumático es un cuadro clínico de difícil manejo. Más aún cuando no existe posibilidad de evacuación del mismo y el paciente en un lapso de tiempo breve se descompensa de todas sus funciones vitales con consecuencias clínicas irreversibles. Existen múltiples alternativas para soportar dichas funciones pero las mismas hacen que el despliego de recursos, (tiempo, dinero, profesionales) sea considerable, configurando de esta forma un perfil del equipo de Enfermería preparada para cualquier situación, dejando sello de su identidad, de sus conocimientos y de sus técnicas.

Recibido: 20 Diciembre 2011

Revisado: 10 Enero 2011

Modificado: 20 Enero 2012

Aceptado: 6 Febrero 2012

\begin{tabular}{|c|c|c|c|c|c|c|c|c|c|c|c|c|c|c|c|}
\hline & Fi02 & $\mathrm{Ph}$ & $\mathrm{Pa} 02$ & $\mathrm{PCO2}$ & Urea & Creatinina & $\mathrm{K}_{+}$ & Htco & $\mathrm{Hb}$ & Leucocitos & TTPA & Plasma & Hematies & Diuresis & Diálisis \\
\hline Día 1 & $21 \%$ & & & & 8,5 & 191 & 3,3 & 36,1 & 12,7 & 16.500 & 33 & - & - & Anuria & - \\
\hline Día 2 & GN $31 x^{\prime}$ & 7,29 & 29 & 47 & 10,6 & 288 & 3,7 & 22,7 & 7,7 & 20.400 & 24 & 2 & 4 & $845 c c$ & - \\
\hline Día 3 & Hudson/VMNI & 7,22 & 35 & 59 & 16,1 & 489 & 3,6 & 24,9 & 8,9 & 18.900 & 22 & - & - & $1225 c c$ & - \\
\hline Día 4 & $\begin{array}{l}\text { VM } 100 \% . \\
\text { PEEP }+8\end{array}$ & 7,19 & 92 & 58 & 19,9 & 641 & 4,2 & 23,5 & 8 & 16.400 & - & - & 4 & $1160 c c$ & - \\
\hline Día 5 & $\begin{array}{l}\text { VM } 80 \% \\
\text { PEEP +8 }\end{array}$ & 7,22 & 74 & 45 & 25 & 716 & 3,8 & 27,7 & 9,2 & 14.700 & 23 & - & - & $1935 c c$ & - \\
\hline Día 6 & $\begin{array}{l}\text { VM } 100 . \\
\text { PEEP }+10\end{array}$ & 7,32 & 78 & 46 & 28,9 & 697 & 2,7 & 25,7 & 8,8 & 12.300 & 24 & - & - & $985 c c$ & - \\
\hline Día 7 & $\begin{array}{l}\text { VM } 100 . \\
\text { PEEP +10 }\end{array}$ & 7,24 & 72 & 51 & 32,7 & 805 & 4 & 30,5 & 10,6 & 11.200 & 24 & - & - & $335 c c$ & - \\
\hline Día 8 & $\begin{array}{l}\text { VM } 100 . \\
\text { PEEP +10 }\end{array}$ & 7,28 & 93 & 42 & 33,6 & 803 & 3,8 & 27,1 & 9,4 & 24.000 & - & - & - & $225 c c$ & HDFVVC \\
\hline Día 9 & $\begin{array}{l}\text { VM } 100 . \\
\text { PEEP +10 }\end{array}$ & 7,21 & 91 & 41 & 15,8 & 314 & 5 & 29 & 10,2 & 35.600 & - & - & - & - & HDFVVC \\
\hline Día 10 & $\begin{array}{l}\text { VM } 100 . \\
\text { PEEP +10 }\end{array}$ & 7,14 & 79 & 55 & 12,1 & 253 & 6,3 & 29,8 & 10,1 & 34.700 & - & - & - & - & HDFVVC \\
\hline \multicolumn{16}{|c|}{$\begin{array}{l}\text { Hudson/ Mascarilla Alta concentración } \\
\text { VM: Ventilación Mecánica } \\
\text { VMNI:Ventilación Mecánica No Invasiva } \\
\text { PEEP: Presión Positva al final de la espiración } \\
\text { HDFVVC: Hemodiafiltración Vena-Vena Continua }\end{array}$} \\
\hline
\end{tabular}

Tabla 1. Evolución Clínico-analítica durante los 10 días de ingreso 


\section{Bibliografía}

1. Andrade PJD y cols. Síndrome de Wunderlich. Hematoma perirrenal espontaneo. Rev Mex Urol 2005; 65(5): 357-362.

2. Flores Cobos A E et al. "Hematoma espontáneo renal y esplénico. Presentación de un caso". Anales de Radiología México 2007;3:239-242.

3. Chao-Yang Chen, et al. Spontaneous Bilateral Renal Subcapsular Hematoma as a Possible Complication of Myeloproliferative Disorders.J Med Sci 2009;29(5):273-275.

4. Castañer Moreno J. Instituto Superior de Medicina Militar Dr. Luis Díaz Soto. "Insuficiencia Renal Aguda Postraumática". Rev Cubana Med Milit 1999;28(1):41-8.

5. Waikar SS, Curhan GC, Wald R, McCarthy EP, Chertow GM. Declining mortality in patients with acute renal failure,1998to 2002. JAmSocNephrol.2006; 17:1143-50. 2.

6. Liaño $F$, Junco E, Pascual J, Madero R, Verde E. The spectrum of acute renal failure in the intensive care unit compared with that seeninothersettings. KidneyIntSuppl.1998;66:S16-24.
7. Sirvent JM, Vallés $M$, .Navas $E$, Calabia J, Ortiz $P$ y Bonet A. "Evolución de los pacientes Críticos con Fracaso Renal Agudo y disfunción multiorgánica tratados con hemodiafiltración venovenosa continua" Med Intensiva.2010;34(2):95-101.

8. García Córdoba $F$, et al. Análisis de las técnicas continuas de sustitución renal en pacientes críticos con insuficiencia renal aguda. Med Intensiva 2002;26(7):349-55.

9. Pannu N, Klarenbach S, Wiebe N, Manns B, Tonelli M. Alberta. Kidney Disease Network. Renal replacement therapy in patients with acute renal failure: A systematic review. JAMA. 2008;299:793-805.

10. Hechavarría Soulary JC, Suárez Domínguez R, Armaignac Ferrer G, Del Pozo Hessing C. Infección nosocomial en la Unidad de Cuidados Intensivos. MEDISAN 2001;5(3):12-17

11. Molano Álvarez E, et al. Enfermería de cuidados críticos y técnicas continuas de reemplazo renal en la Comunidad de Madrid. Enferm Intensiva 2003;14(4):135-147.

12. Canseco Morales J, Luviano García J A, Macías García M T, Beneficios clínicos de la terapia de reemplazo renal continua en el paciente críticamente enfermo con falla renal aguda. Rev Asoc Mex Med Crit y Ter Int 2010;24(2):66-71. 